\title{
Ultrasound-assisted intraoperative localization and laparoscopic management of a previously missed unruptured retroperitoneal ectopic pregnancy
}

\author{
Athanasios Protopapas • Nikolaos Akrivos • Stavros Athanasiou • \\ Ioannis Chatzipapas • Aikaterini Domali • Dimitrios Loutradis
}

Received: 6 February 2014 / Accepted: 29 April 2014 /Published online: 21 May 2014

(C) Springer-Verlag Berlin Heidelberg 2014

\begin{abstract}
Primary retroperitoneal ectopic pregnancy represents an extremely unusual entity with a rather obscure pathogenesis. Implantation in the retroperitoneal space has been reported to occur both spontaneously and with use of assisted reproduction techniques. The pelvic and the upper retroperitoneum have both been involved, and implantation in the most unusual anatomic sites has been reported. The majority of retroperitoneal gestations are located close to large blood vessels, and laparotomy is performed because of the high risk of massive hemorrhage. Few cases have been treated with laparoscopy so far. We report the case of an early firsttrimester retroperitoneal broad ligament live pregnancy occurring after spontaneous conception in a patient who had a history of an ipsilateral tubal ectopic pregnancy, previously treated with laparoscopic right salpingectomy. Current gestation had been missed during initial laparoscopy, and was located and removed during a repeat laparoscopic procedure under intraoperative ultrasonographic guidance.
\end{abstract}

Keywords Retroperitoneal pregnancy - Abdominal pregnancy $\cdot$ Ectopic pregnancy $\cdot$ Laparoscopy

\section{Introduction}

Ectopic pregnancy occurs in $1.5-2 \%$ of all gestations, and is one of the major causes of maternal mortality during the first trimester of pregnancy, accounting for $6 \%$ of all pregnancy-

A. Protopapas $(\bowtie) \cdot$ N. Akrivos $\cdot$ S. Athanasiou $\cdot$ I. Chatzipapas $\cdot$

A. Domali $\cdot$ D. Loutradis

1st University Department of Obstetrics and Gynecology of the

University of Athens, "Alexandra" Hospital, 80 Queen Sophie Ave.,

11528 Athens, Greece

e-mail: prototha@otenet.gr related deaths [1]. Most ectopic pregnancies (95\%) are located in the fallopian tubes, whereas the ovary and abdominal cavity are less frequently involved [1]. Abdominal pregnancy is the rarest form of ectopic pregnancy with an incidence of $1.3 \%$ amongst all ectopics, and mortality rates are seven times higher than in non-abdominal cases [2, 3]. Abdominal pregnancies have been classified as either primary or secondary. Most abdominal pregnancies originate as tubal or ovarian pregnancies that rupture into the peritoneal cavity, where they re-implant [4]. A small fraction of the reported cases occur as a result of primary implantation either in the peritoneal cavity or the retroperitoneum [4].

The occurrence of an ectopic pregnancy in a retroperitoneal location is very rare. In 1938, the incidence of this condition had been reported to be 1 in 183,900 pregnancies [5]. To date, less than 25 well-documented cases of primary retroperitoneal pregnancy implantation have been reported in the medical literature. Development of an ectopic pregnancy in a retroperitoneal location has been reported to occur in the most unusual anatomic sites, such as the rectovaginal space [6], the obturator fossa [7], between the leaves of the broad ligament [8], at the level of the right paracolic sulcus [9], above the inferior vena cava [10], in the upper retroperitoneum [11], and even attached to the head of the pancreas [12]. Both spontaneous conception and assisted reproductive technologies (IUI and IVF-ET) have been implicated in the retroperitoneal development of ectopic pregnancies [6, 12-14]. Gestational age at first diagnosis and clinical presentations may vary considerably, from the asymptomatic woman in her early first trimester of pregnancy to the hemodynamically unstable patient with an advanced ruptured ectopic gestation presenting with lifethreatening retroperitoneal hemorrhage. As a result, management strategies should be tailored to the individual patient. Laparotomy, laparoscopy, and medical treatment with 
methotrexate have all been used in the treatment of retroperitonal pregnancies of various locations.

We report the case of an early first trimester retroperitoneal pregnancy occurring after spontaneous conception in a patient who had a history of an ipsilateral tubal ectopic pregnancy, previously treated with laparoscopic right salpingectomy. The current ectopic was developing between the leaves of the right broad ligament. The living retroperitoneal gestation had been missed during initial laparoscopy, and was located and removed during a repeat laparoscopic procedure under intraoperative ultrasonographic guidance.

\section{Case report}

A 31-year-old woman presented to our department with a 6week history of amenorrhea and a positive pregnancy test for routine antenatal care. Her medical history was unremarkable. Her obstetric history included a right tubal ectopic pregnancy managed by laparoscopic salpingectomy, followed by a term normal vaginal delivery of a healthy infant.

At presentation, the patient was asymptomatic and hemodynamically stable. Transvaginal sonography at $6^{+3}$ weeks, showed an empty uterine cavity. A gestational sac with embryonic heart activity was demonstrated to the right side of the uterus and in contact with the uterine fundus. On clinical examination, no vaginal bleeding was observed, and no lower abdominal or adnexal pain was elicited during bimanual examination. $\beta$-hCG levels were $7,450 \mathrm{mIU} / \mathrm{ml}$, whereas her hemoglobin levels were within normal limits.

With the possible diagnosis of a right cornual pregnancy, the patient was scheduled for laparoscopic evaluation and management. At laparoscopy, the uterus was found slightly enlarged. The fundus was of normal shape and contour. The left adnexa and right ovary were normal. The right tube was found amputated at the level of the isthmus. No evidence of an ectopic intraperitoneal pregnancy was found anywhere in the pelvis, and the procedure was completed without any further intervention.

The following day, a repeat pelvic ultrasound confirmed once more the presence of an ongoing live pregnancy in contact with the right uterine cornu. Furthermore, levels of $\beta$-hCG were rising $(9,832 \mathrm{mIU} / \mathrm{ml})$. Assuming that we had failed to locate the pregnancy during our previous laparoscopy, we decided for a second attempt, and the patient was taken again to the operating theater.

At first, hysteroscopy was performed, but no pregnancy sac was seen in the uterine cavity or near the right ostium. Laparoscopy, this time under ultrasound guidance, followed. Initial laparoscopic findings were again identical to those described above (Fig. 1). With the aid of the transvaginal ultrasound probe, we identified once more the fetal sac to the right of the uterine fundus and managed to locate its exact position below and caudally to the right round ligament by carefully probing with a grasper the anterior leaf of the broad ligament, a maneuver that distorted the ultrasound image of the underlying pregnancy sac.

The round ligament and the anterior leaf of the broad ligament were opened above this area which was infiltrated with diluted vasopressin to reduce blood loss (Figs. 2 and 3). The reproperitoneal space was carefully dissected and a $3 \times$ $2.5 \times 2 \mathrm{~cm}$ bulging mass was identified, arising from the right side of the uterine corpus (Figs. 4 and 5). Further dissection of the mass revealed the presence of a gestational sac (Figs. 6 and 7) through which an intact embryo could be clearly seen (Fig. 8). The sac was opened and the embryo along with the trophoblastic tissue were removed (Figs. 9 and 10). After evacuation of its trophoblastic contents, a fibrous capsule could be clearly identified. This structure had no communication with the uterine cavity (Fig. 11). Hemostasis was accomplished with bipolar diathermy and the broad and round ligaments were reconstructed with interrupted absorbable sutures (Fig. 12).

Our patient made an uneventful recovery and was discharged from our hospital on the second postoperative day. $\beta$-hCG levels were measured weekly and within 4 weeks they had returned to prepregnancy levels.

\section{Discussion}

Retroperitoneal ectopic pregnancy represents an extremely unusual entity with a rather obscure pathogenesis. Its incidence remains largely unknown mainly as a result of the frequent false reporting of abdominal intraperitoneal ectopic gestations with peritoneal invasion, as true retroperitoneal pregnancies. In the case of broad ligament ectopic pregnancy, according to Champion and Tessitore, the anatomical landmarks that surround the ectopic sac should include (a) the uterus medially, (b) the pelvic side walls laterally, (c) the pelvic floor inferiorly, and (d) the uterine tube or round ligament of the uterus superiorly [5]. These were exactly the boundaries in our case. To our opinion, the overlying peritoneum should also be found intact in order to confirm the diagnosis of a true retroperitoneal gestation.

Nevertheless, it is rather difficult to come up with a convincing explanation of how the embryo implanted in the retroperitoneal space in our case, as in others with similar locations. The patient conceived spontaneously and her only uterine surgery was a laparoscopic salpingectomy that preceded a normal-term vaginal delivery. The presence of a very small fistulous tract, resulting from past thermal injury during salpingectomy, cannot be entirely excluded as a causal factor. Nevertheless, the sac was found sufficiently distal to the tubal stump to support such a hypothesis. Another possible explanation, proposed by several investigators, is that the fertilized 
Fig. 1-12 The film of

the procedure
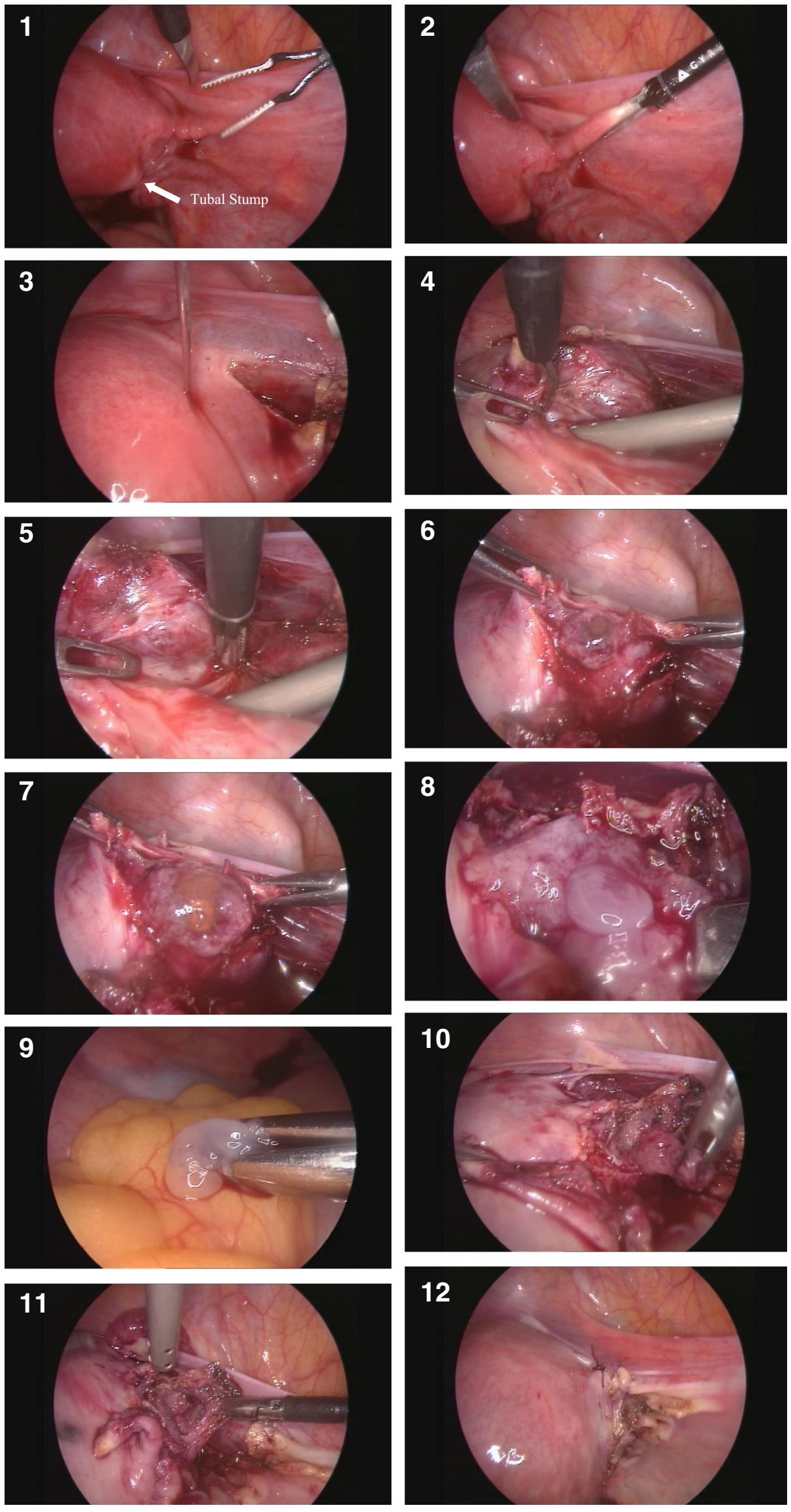

Springer 
ovum may have reached the retroperitoneal space via the lymphatic system [7, 14, 15]. This hypothesis is supported by finding lymphatic tissue with the ectopic mass [15]. The transperitoneal route of implantation of the ectopic to the retroperitoneum through trophoblastic invasion provides a third yet not very convincing mechanism in our case, taking into account three factors: presence of an intact tubal stump, presence of healthy peritoneum above the sac, and conception occurring without use of assisted reproduction techniques.

Assisted reproductive techniques (both IVF-ET and IUI) appear to increase the risk of an ectopic pregnancy and thus implantation at unusual sites, which may be difficult to diagnose and have a high risk of life-threatening complications. Four mechanisms have been suggested for the abdominal location of an ectopic pregnancy in IVF-ET patients: spontaneous retrograde migration of the embryo after intrauterine transfer, iatrogenic placement of embryos in the retroperitoneal space at the time of transfer due to uterine perforation, retroperitoneal implantation through a fistulous tract, and transfer of the embryo from the uterine cavity to the retroperitoneal space through lymphatic channels $[6,7,11]$.

Most of the reported cases of retroperitoneal pregnancies are located close to large blood vessels and the decision to dissect out the gestational tissue should not be taken without appropriate patient preparation and blood bank coverage. In the majority of such cases, laparotomy is performed because of the high risk of massive hemorrhage [11, 12, 16, 17]. The same applies naturally to cases with signs of acute and lifethreatening intra-abdominal bleeding. Laparoscopic management has not been applied frequently, because of the risk of uncontrollable bleeding due to extensive trophoblastic invasion of the retroperitoneal vasculature. The incidence of deep trophoblastic infiltration of large retroperitoneal vessels has not been clearly reported in the existing literature. Nevertheless, there have been few reports of successful laparoscopic management of early retroperitoneal ectopic gestations, such as ours $[3,6,10,18]$, including a case with implantation of the sac on the inferior vena cava [19]. The laparoscopic approach is feasible and should be the treatment of choice, in hemodynamically stable patients without signs of rupture. Before attempting laparoscopic management of such cases, exclusion of large retroperitoneal vascular infiltration with the assistance of MRI may be necessary, especially in more advanced gestations. To our opinion, rupture of a retroperitoneal gestation is a contraindication for laparoscopic management as it results in a difficult to control, narrow operative field due to excessive bleeding from neovascularization. Injection of dilute vasopressin may assist in the dissection of the gestational sac, from surrounding structures, but one has to keep in mind that hemostasis should be meticulous, as the risk of a postoperative hematoma formation is high. Any gynecologist attempting such a procedure should be well-trained, have a thorough knowledge of the retroperitoneal anatomy, and be ready to convert to laparotomy in case of intraoperative complications or uncontrollable bleeding. Close cooperation with a general surgeon and/or an interventional radiologist may prove invaluable to safely conclude these procedures.

Adjuvant treatment with methotrexate, systemic or through selective arterial embolization has been suggested to control the risk of bleeding from the placental bed and to avoid the possibility of persistent trophoblastic tissue [3, 20]. Although surgery remains the mainstay of treatment for abdominal ectopic pregnancies, there are also case reports of early abdominal pregnancies being treated successfully with systemic methotrexate, leading to its resorption without the need for further surgery [21]. Factors that are associated with failure of medical management include initial $\beta$-hCG values greater than $5,000 \mathrm{mUI} / \mathrm{mL}$, ultrasound detection of a moderate or large amount of free peritoneal fluid, the presence of fetal cardiac activity, and a pretreatment increase in the $\beta$-hCG level of more than $50 \%$ over a 48 -h period $[1,3,5]$. Our case presented with three out of four of the above-mentioned contraindications for medical management. Furthermore, the patient was hemodynamically stable, and this permitted the use of the transvaginal probe to assist in the exact localization of the ectopic gestation. Other preoperative imaging techniques, and in particular magnetic resonance imaging (MRI), may prove useful in guiding operative maneuvers but they are costly and not always readily available. It is very probable that the second laparoscopy would have been avoided if we had used intraoperative ultrasound during first surgery. We decided not to administer systemic methotrexate postoperatively, as removal of the trophoblastic tissue appeared complete. Indeed levels of $\beta$-hCG declined steeply postoperatively, indicating its complete excision.

In conclusion, although retroperitoneal pregnancy is an extremely rare condition, in a patient with clinical findings suggestive of ectopic pregnancy, if both the uterus and adnexa are normal during laparoscopic exploration, unusual locations such as the retroperitoneum should be carefully examined. Ipsilateral or bilateral salpingectomy does not exclude the occurrence of a parametrial pregnancy, and a clinician should be aware of such a possibility. Ultrasound should be used intraoperatively especially when we are dealing with a small and difficult-to-locate parametrial pregnancy

Informed consent All procedures followed were in accordance with the ethical standards of the responsible committee on human experimentation (institutional and national) and with the Helsinki Declaration of 1975 , as revised in 2000. Informed consent was obtained from all patients for included in the study.

Conflict of interest All authors declare no conflict of interest. 


\section{References}

1. Barnhart KT (2009) Ectopic pregnancy. N Engl J Med 361:379-387

2. Chetty M, Elson J (2009) Treating non-tubal ectopic pregnancy. Best Pract Res Clin Obstet Gynaecol 23:529-538

3. Tsudo T, Harada T, Yoshioka H, Terakawa N (1997) Laparoscopic management of early primary abdominal pregnancy. Obstet Gynecol 90:687-688

4. Lee JW, Sohn KM, Jung HS (2005) Retroperitoneal ectopic pregnancy. Am J Reprod 184:1600-1601

5. Champion PK, Tessitore NJ (1938) Intraligamentary pregnancy: a survey of all published cases of over 7 calendar months, with the discussion of an additional case. Am J Obstet Gynecol 36:281-293

6. Martinez-Varea A, Hidalgo-Mora JJ, Paya V, Morcillo I, Martin E, Pellicer A (2011) Retroperitoneal ectopic pregnancy after intrauterine insemination. Fertil Steril 95:2433e1-e3

7. Lin JX, Liu Q, Ju Y, Guan Q, Wu YZ, Zheng N (2008) Primary obturator foramen pregnancy: a case report and review of literature. Chin Med J 121:1328-1330

8. Abdul MA, Tabari AM, Kabiru D, Hamidu N (2008) Broad ligament pregnancy: a report of two cases. Ann Afr Med 7(2):86-87

9. Chang YL, Ko PC, Yen CF (2008) Retroperitoneal abdominal pregnancy at left parcolic sulcus. J Minim Invasive Gynecol 15:660-661

10. Bae SU, Kim CN, Hwang IT, Choi YJ, Lee MK, Cho BS, Kang Y, Park JS (2009) Laparoscopic treatment of early retroperitoneal abdominal pregnancy implanted on inferior vena cava. Surg Laparosc Endosc Percut Tech 19(4):e156-e158

11. Ferland RJ, Chadwick DA, O'Brien JA, Granai CO (1991) An ectopic pregnancy in the upper retroperitoneum following in vitro fertilization and embryo transfer. Obstet Gynecol 78:544-546

12. Dmowski WP, Rana N, Ding J, Wu WT (2002) Retroperitoneal subpancreatic ectopic pregnancy following in vitro fertilization in a patient with previous bilateral salpingectomy: how did it get there? J Assist Reprod Genet 19(2):90-93

13. Apantaku O, Rana P, Inglis T (2006) Broad ligament ectopic pregnancy following in-vitro fertilisation in a patient with previous bilateral salpingectomy. J Obstet Gynaecol 26(5):474

14. Iwama H, Tsutsumi S, Igarashi H, Takahashi K, Nakahara K, Kurachi $\mathrm{H}$ (2008) A case of retriperitoneal ectopic pregnancy following IVFET in a patient with previous bilateral salpingectomy. Am J Perinatol 25:33-36

15. Rersson J, Reynisson P, Masback A, Epstein E, Saldeen P (2010) Histopathology indicates lymphatic spread of a pelvic retroperitoneal ectopic pregnancy removed by robot-assisted laparoscopy with temporary occlusion of the blood supply. Acta Obstet Gynecol Scand 89(6):835-839

16. Hall JS, Harris M, Levy RC, Walrond ER (1973) Retroperitoneal ectopic pregnancy. J Obstet Gynaecol Br Commonw 80:92-94

17. Siow A, Chern B, Soong Y (2004) Successful laparoscopic treatment of an abdominal pregnancy in the broad ligament. Singap Med J 45(2):88-89

18. Olsen ME (1997) Laparoscopic treatment of intraligamentous pregnancy. Obstet Gynecol 89:862

19. Bae SU, Kim CN, Kim KH, Hwang IT, Choi YJ, Lee MK, Cho BS, Kang YJ, Park JS (2009) Laparoscopic treatment of early retroperitoneal abdominal pregnancy implanted on inferior vena cava. Surg Laparosc Endosc Percutan Tech 19(4):e156e158

20. Parant O, Sarramon MF, Laffitte A, el Ghaoui A, Reme JM (1999) Parametrial pregnancy. Report of a case of "paracervical" pregnancy treated by medico-surgical management. J Gynecol Obstet Biol Reprod (Paris) 28(1):69-72

21. Okorie CO (2010) Retroperitoneal ectopic pregnancy: is there any place for non-surgical treatment with methotrexate? J Obstet Gynaecol Res 36(5):1133-1136 